\title{
Limited prosthesis wear in geriatric diabetic edentulous patients: A social morbidity
}

\author{
Manu Rathee, Amit Tamrakar ${ }^{1}$ \\ Departments of Prosthodontics, Post Graduate Institute of Dental Sciences, Pandit Bhagwat Dayal Sharma University of Health Sciences, Rohtak, \\ Haryana, 'Jamia Milia Islamia, New Delhi, India
}

\section{A B S T R A C T}

Tooth loss is a widespread geriatric healthcare issue.The problems with the complete denture wear in edentulous patients are compounded by the coexisting diabetes. The dryness of thin oral mucosa resultant from diabetes or as side effect of medications used for various advanced age health problems lead to restricted prosthesis wear. Impaired chewing ability and denture problems can cause isolation among geriatric population.

Key words: Complete denture, Denture wear, Diabetes, Halitosis

Edentulism continues to be a global health care issue that represents as an increase in demand of complete dentures due to increased number of elderly persons in the society. Tooth loss is inevitable with advancing age and commensurate with tooth loss is the need to have removable dentures. ${ }^{[1]}$ Common complaints by elderly people with dental prosthesis, particularly removable complete dentures include difficulty in eating mainly hard consistency foods and avoidance of social interaction influencing their Quality of Life. ${ }^{[2]}$ Many denture wearers frequently do not wear their dentures that do not fit well. ${ }^{[1]}$ Chewing ability is essential for maintaining social life and denture problems can lead to social isolation.

Geriodontic problems are considered to be caused by physiological changes and intensified by common diseases. ${ }^{[3]}$ Completely edentulous patients are observed to be at higher risk of being diabetic (odd ratio 1.82). ${ }^{[4 \mid}$ The prevalence of dry mouth and hyposalivation, exaggerated

\begin{tabular}{|l|l|}
\hline \multicolumn{2}{|c|}{ Access this article online } \\
\hline Quick Response Code: & Website: \\
\hline & www.joshd.net \\
\hline & \\
\hline
\end{tabular}

by disease and medication, is found to be high in geriatric diabetics causing pain and discomfort. ${ }^{[5]}$ The awareness of diabetic patients about their increased risk for oral diseases is low and the attitude towards maintenance of good oral health is also poor ${ }^{[6]}$ Maintaining oral and dental hygiene is challenging for geriatric population due to limited motor skills.

Halitosis or malodor refers to any unpleasant odor emerging from the mouth that is detected by others and it negatively influences socialization. Poor oral hygiene and dry mouth are common oral causes of halitosis. ${ }^{[7]}$ Unclean dentures, lower educational level, attitude towards prosthesis care, hyposalivation and wearing dentures during night are the factors that are related to oral malodor. ${ }^{[8]}$ Women are observed to be more aware of halitosis than men. ${ }^{[2]}$ Denture adhesives are differentially active against oral malodor related microbs. ${ }^{[9]}$

Diabetic microangiopathy can cause thinning of oral mucous membrane, lowered immune response and reduced salivary flow leading to xerostomia. ${ }^{[10]}$ Aging is the main cause of xerostomia and aging mucosa is influenced by harmful effects of Diabetes Mellitus. ${ }^{[5,10]}$ Increased virulence and increased adherence of microorganism in high glucose levels makes the oral mucosa prone to opportunistic microbial invasion. Denture bearing mucosa is subjected to stress and trauma that is adversely

Corresponding Author: Dr. Manu Rathee, Senior Professor and Head, Post Graduate Institute of Dental Sciences, Pandit Bhagwat Dayal Sharma University of Health Sciences, Rohtak, Haryana, India. E-mail: ratheemanu@gmail.com 
influenced by mal directional occlusal load, uneven wear of artificial resin teeth, lost vertical dimension of occlusion and thermal injury. ${ }^{[10]}$ Complete denture wearers commonly have candidal colonization of denture mucosa, exacerbated in uncontrolled diabetes. ${ }^{[1]}$ The increase in oral candidiasis is positively correlated with elevated blood glucose level and showed no variation in pattern of colonization between the genders.

It is important to include dentist and hygienist in the team for care of elderly. Regular dental checkups and oral care maintenance can help delay edentulousness. ${ }^{[5]}$ The patients with dry mouth and sensitive mucosa should avoid using products containing flavoring agents and detergents. ${ }^{[11]}$ Xerostomic elderly can be managed by frequent sipping of water or milk, salivary substitutes, avoiding dry foods, regular dental visits and improved oral hygiene. The inflammatory response in mucosa underneath complete denture is greatly reduced when diabetes is brought under control. The oral candidal colonization in complete denture wearers can be checked by controlling the blood glucose levels in uncontrolled diabetics with oral hypoglycemic drugs. There is a need to develop programs to educate the society to spread awareness about oral implications of diabetes and its relation with oral health particularly and systemic health in general.

The high prevalence of halitosis supports the fact that the public awareness is to be increased about daily hygiene measures for mouth and dental prosthesis as despite widespread nature of problem, public's knowledge about this condition is poor. ${ }^{[12]}$ Oral hygiene instructions regarding daily hygiene measures for maintaining a clean oral cavity can help reduce halitosis. Daily brushing the tongue lowers the microbial plaque on the surface of the tongue ${ }^{[8]}$ Removing the dentures daily night and regularly cleaning it properly, may be with assistance from other person, is advisable to prevent halitosis and promote socialization. All efforts need to be directed towards minimizing denture problems, promoting oral health that reflects in improved socialization.

\section{REFERENCES}

1. SavocaMR, Arcury TA, LengX, Chen H, Bell RA,Anderson AM, et al. Impact of denture usage patterns on dietary quality and food avoidance among older adults. J Nutr Gerontol Geriatr 2011;30:86-102.

2. Bekiroglu N, Çiftçi A, Bayraktar K, Yavuz A, Kargul B. Oral complaints of denture-wearing elderly people living in two nursing homes in Istanbul, Turkey. Oral Health Dent Manag 2012;11:107-15.

3. Rai S, Kaur M, Goel S, Bhatnagar P. Moral and professional responsibility of oral physician toward geriatric patient with interdisciplinary management - The time to act is now! J Midlife Health 2011;2:18-24.

4. Felton DA. Edentulism and comorbid factors. Tex Dent $\mathrm{J}$ 2010;127:389-401.

5. Kossioni AE, Dontas AS. The stomatognathic system in the elderly. Useful information for the medical practitioner. Clin Interv Aging 2007;2:591-7.

6. Eldarrat $\mathrm{AH}$. Awareness and attitude of diabetic patients about their increased risk for oral diseases.Oral Health Prev Dent 2011;9:235-41.

7. Pratibha PK, Bhat KM, Bhat GS. Oral malodor: A review of the literature. J Dent Hyg 2006;80:8.

8. Garrett NR. Poor oral hygiene, wearing dentures at night, perceptions of mouth dryness and burning, and lower educational level may be related to oral malodor in denture wearers. J Evid Based Dent Pract 2010;10:67-9.

9. Polyzois G, Stefaniotis T, Papaparaskevas J, Donta C. Antimicrobial efficacy of denture adhesives on some oral malodorrelated microbes. Odontology 2013;101:103-7.

10. Ganapathy DM, Joseph S, Ariga P, Selvaraj A. Evaluation of the influence of blood glucose level on oral candidal colonization in complete denture wearers with Type-II Diabetes Mellitus: An in vivo Study. Dent ResJ (Isfahan) 2013;10:87-92.

11. Hitz Lindenmüller I, Lambrecht JT. Oral care.Curr Probl Dermatol 2011;40:107-15.

12. Mbodj EB, Faye B, Faye D, Seck MT, Sarr M, Ndiaye C, et.al. Prevalence of halitosis in patients with dental prostheses in Senegal.Med Trop (Mars) 2011;71:272-4.

How to cite this article: Rathee M, Tamrakar A. Limited prosthesis wear in geriatric diabetic edentulous patients: A social morbidity. J Soc Health Diabetes 2014;2:112-3.

Source of Support: Nil. Conflict of Interest: None declared. 